\title{
Folk Intuitions, Science Fiction and Philosophy: Comment on Experimental Philosophy
}

\author{
Renia Gasparatou* \\ DESECE, School of Humanities \& Social Sciences, \\ University of Patras, Rion 26500, Greece \\ * E-mail: gasparat@upatras.gr
}

\begin{abstract}
Some experimental philosophers imply that philosophers should endorse folk intuitions and even use them to advance philosophical theses. In this paper I will try to contrast experimental appeals to intuition with J. L. Austin's, whom some experimentalists cite as a precursor of their method. I will suggest that Austin evokes ordinary intuitions in order to dismantle philosophical quests. He even suggests (a) that the appeal to ordinary intuitions of the folk can hardly prescribe answers to extraordinary circumstances and (b) that philosophical quests themselves are extraordinary. Therefore, the appeal to folk intuitions cannot prescribe answers to philosophical problems. It can only dissolve philosophical quests; yet, the dismantling of philosophy hardly accords with the experimentalists' agenda.
\end{abstract}

\section{Keywords}

Experimental philosophy, J. L. Austin, intuition

Many philosophers defend reasoning by appealing to intuition: Philosophy is a conceptual investigation; the use of concepts imposes conceptual rules to all competent speakers; philosophers are entitled, being competent speakers themselves, to invoke their intuitions concerning some target concept and analyse them. Intuitions, thus, provide us with the epistemic standards of our community (see also Gasparatou, 2009).

Today experimental philosophy challenges this methodology; it uses empirical methods to investigate whether people's intuitions are univocal and to determine what factors may influence them. Laypersons are asked to report their intuitions in relation to some hypothetical scenario. The story is somehow associated to a philosophical problem and is supposed to trigger philosophically interesting intuitions. Such experiments show that factors such as one's background knowledge, beliefs, culture, intellectual habits, emotions, even attendance of philosophy courses, can influence our intuitions. Thus, they 
do not arise solely from conceptual norms; nor are they as unanimous as expected. Studies that move along those lines criticise the traditional armchair appeal to intuition. Philosophers cannot represent the folk for their intuitions differ. So they cannot just sit on their armchairs and use their own intuitions to support general theories.

However, some experimentalists do not disregard the appeal to intuition completely. They imply that philosophers should endorse the community's intuitions since many philosophical problems arise from common sense (for comprehending introductions to experimental philosophy, see Alexander and Weinberg, 2007; Knobe and Nichols, 2008).

Yet, some of those studies ask the folk to consider highly extraordinary stories: Nahmias et al. $(2005,2008)$ challenge the claim that incompatibilism is intuitive and discuss the philosophical significance of their findings. Incompatibilists believe free will is impossible if determinism is true; they often claim that this view is supported by commonsensical intuitions (see, for example, Strawson, 1986; Kane, 1999). In order to investigate that claim, Nahmias et al. asked a pool of participants to deliberate on the following story:

Imagine that in the next century we discover all the laws of nature, and we build a supercomputer which can deduce from these laws of nature and from the current state of everything in the world exactly what will be happening in the world at any future time. It can look at everything about the way the world is and predict everything about how it will be with $100 \%$ accuracy. Suppose that such a supercomputer existed, and it looks at the state of the universe at a certain time on March 25*, 2150 A.D., twenty years before Jeremy Hall is born. The computer then deduces from this information and the laws of nature that Jeremy will definitely rob Fidelity Bank at 6:00 PM on January 26'h, 2195. As always, the supercomputer's prediction is correct; Jeremy robs Fidelity Bank at 6:00 PM on January $26^{\circ} \mathrm{h}, 2195$.

Do you think that, when Jeremy robs the bank, he acts of his own free will?

A significant majority (76\%) of participants judged that Jeremy acts of his own free will. They also examined the possibility that participants were influenced by the negative nature of the action: Nahmias et al. replaced Jeremy's robbing the bank with a positive action (saving a child) for another set of participants and a neutral action (going jogging) for a third set. Changing the moral nature of the action had no significant effect on the participants responses: $68 \%$ judged that Jeremy saves the child of his own free will, and $79 \%$ judged that he goes jogging of his own free will (Nahmias et al., 2005: 565, 2008: 86). 
Nahmias et al. suggest that the folk intuitions regarding free will, which the Jeremy- thought experiments triggered, reveal that the folk (a) think that free will and responsibility are compatible with determinism, hence (b) challenge philosophers' claim that incompatibilism is intuitive, and (c) provide an evidential advantage to compatibilistic philosophical theories:

... a theory... that accords with those [folk] intuitions..., has, all else being equal, a theoretical advantage over a theory that demands revision or elimination of such intuitions. (Nahmias et al. 2008: 85)

Folk intuitions work as a guide to common sense and, according to some experimentalists, problems concerning free will are a clear instance of a puzzle that has arisen from our commonsensical views about human action (Knobe and Nichols, 2008: 8). Thus, studies concerning free will started in order to confront the armchair intuitions of incompatibilist philosophers like Kane, Strawson, etc. But now the list is growing longer and longer; experiments are interpreted alternatively as either allying with compatibilism (Turner and Nahmias, 2006; Nahmias, 2006) or some forms of incompatibilism (Nichols, 2004). For example, Nahmias uses both Nichols studies, as well as some of its own, to suggest that the folk may see reductionism as incompatible with free will, but they do not consider that determinism is incompatible with it. He bravely concludes:

Well, what else would you expect from a compatibilist? I'm just trying to sell my own intuitions like any good philosopher.... (Nahmias, 2006: 234)

The free will debate, as conducted within the community of experimental philosophy, shows how experimental methods are used in order to promote different theories as privileged by common sense. It is not my point here to enter the free will debate but rather to comment on how experimentalists use intuitions. Of course, they do not claim that such theories are true or correct because common sense allies with them (Nahmias et al., 2008: 97). Yet it seems those theories are expected to have some sort of advantage over alternative ones. Folk intuitions supposedly provide a starting point when reasoning about this problem. Moreover, they can help us choose between alternative theses; at the very least, they put the burden of proof to the views opposing common sense (Nahmias et al., 2005, 2008). Commonsensical intuitions then are relevant to philosophy.

Yet, the experiment with Jeremy above, for example, describes a sciencefiction scenario, which has never arisen in ordinary life. Laypersons are asked 
to imagine a different universe in which all the laws of nature have been discovered and some supercomputer can look at everything about the way the world is and predict everything about how it will be with $100 \%$ accuracy. However, it is very difficult to intuitively prescribe whether Jeremy robs the bank of his own free will when the context is out of the ordinary; or when our beliefs are being questioned (see Fodor, 1964; Dennett, 1991: 190). One cannot appeal to common sense while challenging it.

Interestingly enough, some experimentalists regard J. L. Austin as a precursor of their method (Alexander and Weinberg, 2007: 18). He appealed to the commonsensical intuitions which ordinary language triggered; he explicitly defended this method (Austin, 1961: 185 and passim) and was the first to suggest that philosophers should abandon their armchairs and do field work (Austin, 1961: 183). Austin, however, writes:

\begin{abstract}
Suppose you live in harmony and friendship for four years with a cat: and then it delivers a philippic. We ask ourselves, perhaps, 'is it a real cat? Or is it not a real cat?' 'Either it is or it is not but we can't be sure which.' Now, actually, that is not so: neither 'it is a real cat' nor 'it is not a real cat' fits the facts semantically.... With sound instinct, the plain man turns in such cases to Watson and says 'Well, now, what would you say?" How would you describe it? The difficulty is just that: there is no sort of description, which is not misleading... Ordinary language breaks down in extraordinary cases. (Austin, 1961: 67)
\end{abstract}

Austin, thus, claims that one cannot appeal to ordinary intuitions when the situation is extraordinary. Extraordinary refers to whatever does not belong to our existing speech acts, or to whatever situations do not normally occur in everyday life. But there is also a more specific level of extraordinariness that is associated with any effort to go beyond the phenomena to an underlying system of explanation.

Hence, Austin implies that philosophy itself is extraordinary insomuch as it seeks for such explanations; his attempt to prevent that very step when one passes from practical worries into theoretical general questions demonstrates this. The majority of his work is focused around an attempt to "dismantle" philosophical doctrines "before (they) get off the ground" (Austin, 1964: 142). Commonsensical intuitions detected by ordinary language are adequate for all "everyday or practical or ordinary purposes". And this is the uttermost anyone should utilise ordinary intuitions (Austin, 1964: 119); asking more general questions makes no sense. Austin implores commonsensical intuitions specifically because he wants to show that philosophical problems hardly make any sense. 
Dismantling philosophy though, does not seem to be in the experimentalists' agenda. Quite the contrary: they rely on their new empirical methods to impose such questions on common sense and suggest that the intuitions they collect are philosophically interesting. Which, in turn, implies that the extraordinariness of the story described in Nahmias et al. $(2005,2008)$ above is only part of the problem. The crucial difficulty is that experimentalists take the folk's responses to this story as an answer to the philosophical question about incompatibilism. This poses a problem not only for those who describe extraordinary scenarios in their experiments, but for everyone who implies that common sense can take a stand to a philosophical problem. Folk intuitions cannot vote pro or against compatibilism, because this question does not arise in ordinary life. When experimental philosophers take laypersons answers about whether Jeremy acted of his own free will, and interpret them as supporting either philosophical theory, they manipulate common sense to fit their philosophical pronouncements.

It is reasonable to say that the ordinary use of terms should - up to a point be respected. Common sense might be philosophy's starting point; this does not entail it can answer philosophical questions though. In fact, as soon as the philosophical treatment of any problem starts, common sense is challenged. Even if a sceptical doubt sprung after one's dreaming experience in everyday life, the moment she will start worrying about how we can establish what we know about the world is the moment when ordinary language can no longer be of any help. At that very moment she is being transformed into a philosopher; she starts questioning and stops relying on her ordinary intuitions. The only way that an appeal to ordinary intuitions can make sense in such a case is only if one attempts to stop her from doing philosophy: they can suggest, just like Austin, that such a worry (ordinarily) does not make any sense. Since ordinary intuitions cannot answer such questions, the questions themselves do not make sense.

Philosophers who have defended reasoning by appeal to intuition rely on intuitions to unravel conceptual rules and claim they use them mostly to treat philosophical worries; not to address them. Experimentalists however, would share neither claim. They have shown that intuitions do not arise from conceptual rules alone. They suggest that commonsensical intuitions are philosophically interesting on their own merit and even imply they can be utilised to advance their theoretical theses. Yet they need to justify how or why ordinary intuitions can help explore extraordinary questions. 


\section{Acknowledgement}

I would like to thank the State Scholarships Foundation of Greece (IKY) for supporting my post-doc research on the Epistemic Value of Intuitions. This paper is part of this research programme.

\section{References}

Alexander, J. and Weinberg J. M. (2007). Analytic Epistemology and Experimental Philosophy. Philosophy Compass 2, 56-80.

Austin, J. L. (1961). Philosophical Papers. Oxford University Press, Oxford. (1964). Sense and Sensibilia. Oxford University Press, Oxford.

Dennett, D. C. (1991). Consciousness Explained. Little, Brown, Boston, MA.

Fodor, J. (1964). On knowing what we would say. Philosophical Review 73, 198-212.

Gasparatou, R. (2008). Armchair versus Questionnaire Polled Intuitions: Intuitions nevertheless. The Reasoner 2 (11): 7-9.

. (2009). High-standard Epistemology and the Appeal to Intuition. Filozofia 64, 680-692.

Kane, R. (1999). Responsibility, Luck and Chance: Reflections on Free Will and Indeterminism. Journal of Philosophy 96, 217-240.

Knobe, J. and Nichols, S. (2008). An Experimental Philosophy Manifesto. In Knobe, J. and Nichols, S. (Eds) Experimental Philosophy, pp. 3-17. Oxford University Press, Oxford.

Nadelhoffer, T. and Nahmias, E. (2007). The Past and Future of Experimental Philosophy. Philosophical Explorations 10, 123-149.

Nahmias, E. (2006). Folk Fears about Freedom and Responsibility: Determinism vs Reductionism. Journal of Cognition and Culture 6, 215-237.

Nahmias, E., Morris, S., Nadelhoffer, T. and Turner, J. (2005). Surveying Freedom: Folk Intuitions about free will and moral responsibility. Philosophical Psychology 18, 561-584.

- (2008). Is Incompatibilism Intuitive? In Knobe, J. and Nichols, S. (Eds) Experimental Philosophy, pp. 81-104. Oxford University Press, Oxford.

Nichols, S. (2004). The Folk Psychology of Free Will: Fits and Starts. Mind and Language 19, 473-502.

Strawson, G. (1986). Freedom and Belief. Oxford University Press, Oxford.

Turner, J. and Nahmias, E. (2006). Are the Folk Agent-Causationists? Mind and Language 21, 597-609. 